\section{Early stopping in clinical PET studies: How to reduce expense and exposure}

\author{
Jonas E Svensson ${ }^{1,2}$ (1), Martin Schain ${ }^{3}$, Gitte M Knudsen ${ }^{3,4}$, \\ R Todd Ogden ${ }^{5,6}$ and Pontus Plavén-Sigray ${ }^{1,2,3}$ (1)
}

Journal of Cerebral Blood Flow \& Metabolism

202I, Vol. 4I(II) 2805-28I9

(C) The Author(s) 2021

\section{(c) (1)}

Article reuse guidelines: sagepub.com/journals-permissions DOI: $10.1177 / 0271678 \times 211017796$ journals.sagepub.com/home/jcbfm (SAGE

\begin{abstract}
Clinical positron emission tomography (PET) research is costly and entails exposing participants to radioactivity. Researchers should therefore aim to include just the number of subjects needed to fulfill the purpose of the study. In this tutorial we show how to apply sequential Bayes Factor testing in order to stop the recruitment of subjects in a clinical PET study as soon as enough data have been collected to make a conclusion. By using simulations, we demonstrate that it is possible to stop a study early, while keeping the number of erroneous conclusions low. We then apply sequential Bayes Factor testing to a real PET data set and show that it is possible to obtain support in favor of an effect while simultaneously reducing the sample size with $30 \%$. Using this procedure allows researchers to reduce expense and radioactivity exposure for a range of effect sizes relevant for PET research.
\end{abstract}

\title{
Keywords
}

Early stopping, sequential testing, Bayes factor, positron emission tomography, tutorial

Received I December 2020; Revised I3 April 202I; Accepted I8 April 202 I

\section{Introduction}

Positron emission tomography (PET) examinations are expensive and may impose a substantial burden on research budgets. Depending on the local PET centers finances and the experimental design, it is not unusual that researchers pay 5000 Euro/USD or more for a PET-scan of a single subject. In addition to the high cost, a PET scan entails exposing individuals to radioactivity, with average doses often ranging between 0.6 to $5 \mathrm{mSv}{ }^{1}$ It is hence in the interest of the PET researcher to keep the number of included research subjects to a minimum, while still performing enough PET examinations to be able to draw appropriate conclusions from the collected data.

Traditionally, the number of included subjects in a clinical PET study is determined a priori based on a power analysis and the null-hypothesis significance testing (NHST) procedure. ${ }^{2}$ However, a difference between groups may sometimes be detectable before the study reaches the a priori determined sample size. Ideally, the researchers should include just the number of individuals needed to be able to interpret the results with high confidence, no more, no less. When performing more scans than needed, PET researchers are wasting money and exposing people unnecessarily to radioactivity.

One way to avoid superfluous PET scans is to intermittently check for a statistical effect while the study is still ongoing, generally termed sequential testing of data. In the uncorrected NHST framework, however, sequential testing does pose a problem, as it can greatly inflate the nominal false positive rate. $^{3}$ If a new

\footnotetext{
'Centre for Psychiatry Research, Department of Clinical Neuroscience, Karolinska Institutet, Stockholm, Sweden

${ }^{2}$ Stockholm Health Care Services, Region Stockholm, Karolinska University Hospital, Stockholm, Sweden

${ }^{3}$ Neurobiology Research Unit, Copenhagen University Hospital,

Copenhagen, Denmark

${ }^{4}$ Institute of Clinical Medicine, University of Copenhagen, Copenhagen, Denmark

${ }^{5}$ Department of Biostatistics, Mailman School of Public Health, Columbia University, New York, NY, USA

${ }^{6}$ Molecular Imaging and Neuropathology Area, New York State

Psychiatric Institute, New York, NY, USA
}

\section{Corresponding author:}

Pontus Plavén-Sigray, Neurobiology Research Unit 6-8, Inge Lehmanns Vej Rigshospitalet, building 8057, DK-2100 Copenhagen, Denmark.

Email: pontus.plaven-sigray@ki.se 
uncorrected p-value is calculated and used for making inference after the collection of each research subject, the false positive rate will be above $20 \%$ for commonly seen sample sizes in PET literature. ${ }^{4}$

There exist however statistical techniques that are appropriate to use when performing sequential testing. One possibility, called Pocock's correction, ${ }^{5}$ involves lowering the significance threshold for each test so that the overall error rate does not surpass a pre-set rate of false positives (usually 5\%). Another efficient and flexible method is to apply so called alphaspending functions (using e.g., the ÓBrien and Flemming boundaries), where the significance threshold changes for each sequential test, making it harder to reject the null-hypothesis $\left(\mathrm{H}_{0}\right)$ at an early stage of the study, but easier later on, while still allowing for exact control over the rate of false positives. ${ }^{6,7}$

An alternative possibility is to use an entirely different metric to test scientific hypotheses: the Bayes Factor (BF) ${ }^{8-11}$ The BF has been gaining traction in the field of biomedicine during the last decade and has two important characteristics; first, it is well suited for sequential testing of data, ${ }^{12,13}$ and second, it also allows for quantification of relative evidence in favour of $\mathrm{H}_{0}$, meaning that a PET study can be stopped when it is determined that $\mathrm{H}_{0}$ is supported. ${ }^{14}$ In this tutorial we will show how to use sequential BF tests in common PET study designs, in order to stop data collection in a study early.

\section{Bayes factor - A versatile alternative for testing hypotheses}

Support in data for competing hypotheses. Bayesian hypothesis testing using BF aims to assess how compatible the observed data (such as a patient-control difference) is with each of two competing hypotheses. These hypotheses are often specified as the null hypothesis $\left(\mathrm{H}_{0}\right)$ and an alternative hypothesis $\left(\mathrm{H}_{1}\right)$. The null-hypothesis typically states that the effect is exactly zero and the alternative hypothesis states that the effect is different from zero. The BF, quantifying the support in data in favor of the alternative hypothesis over the null-hypothesis, is defined as the likelihood ratio of the two hypotheses

$$
B F=P\left(D \mid H_{1}\right) / P\left(D \mid H_{0}\right)
$$

where D denotes the observed data. ${ }^{15}$ For example, a BF of 4 can be interpreted as "the observed data is 4 times more likely to have occurred under the alternative hypothesis compared to the null hypothesis." As such, the BF directly quantifies the evidence in data in favour of one hypothesis against another. The reciprocal of BF, quantifies the support in data in favour of the null hypothesis, compared to the alternative. A BF of $1 / 5$ would hence mean that there is 5 times more support in the data in favor of $\mathrm{H}_{0}$, compared to $\mathrm{H}_{1}$. See Supplementary material for a more in-depth explanation on the BF, and how it is connected to Bayes theorem.

Evidence thresholds. The BF quantifies evidence on a continuous scale ranging from 0 to infinity, where values over 1 support the hypothesis in the numerator, and values below 1 support the hypothesis in the denominator. A set of thresholds have been suggested to help with the interpretation and decision making when using $\mathrm{BF}^{10}$ (Table 1). A BF of 3 , which often corresponds to a p-value around $0.05^{14,16,17}$ is commonly interpreted as providing "moderate" evidence in favour of one hypothesis over another. It is often considered to be the minimal threshold for claiming support of a hypothesis.

Specifying the alternative hypothesis. In the classical NHST framework, the alternative hypothesis is often specified as meaning any other value than point zero. The use of $\mathrm{BF}$ does however require the researchers to be more specific when describing $\mathrm{H}_{1}$. For example, the researcher could specify the alternative hypothesis as a single value different from zero, such as predicting that a mean patient-control difference in binding potential will be exactly 0.5 . However, since it is rare that researchers are confident in predicting a single point value, the alternative hypothesis is often specified as a probability distribution covering a range of values instead. In doing so, the researchers are "hedging their bets" by spreading the prediction out across many plausible values of an effect. This probability distribution can be informative, ${ }^{18,19}$ e.g. a narrow normal distribution centered around a specific value. It can also be made "non-informative," e.g. a wide distribution centered around zero.

A commonly used non-informative distribution for describing $\mathrm{H}_{1}$ when testing mean differences is a twosided Cauchy distribution centered around zero ${ }^{20}$ (Figure 1, see Supplementary material for a mathematical expression of the Cauchy). This distribution ranges

Table I. Evidence thresholds for the Bayes Factor.

\begin{tabular}{ll}
\hline Bayes Factor & Interpretation \\
\hline$>10$ & Strong evidence in favor of $\mathrm{H}_{1}$ \\
$3-10$ & Moderate evidence in favor of $\mathrm{H}_{1}$ \\
$\mathrm{I}-3$ & Negligible to weak evidence in favor of $\mathrm{H}_{1}$ \\
$\mathrm{I} / 3-\mathrm{I}$ & Weak to negligible evidence in favor of $\mathrm{H}_{0}$ \\
$\mathrm{I} / 10-1 / 3$ & Moderate evidence in favor of $\mathrm{H}_{0}$ \\
$<\mathrm{I} / 10$ & Strong evidence in favor of $\mathrm{H}_{0}$ \\
\hline
\end{tabular}

These are suggested BF threshold for interpreting relative evidence in the data in favor of one hypothesis over another.

Source: Adapted from Lee and Wagenmakers (2014). ${ }^{10}$ 


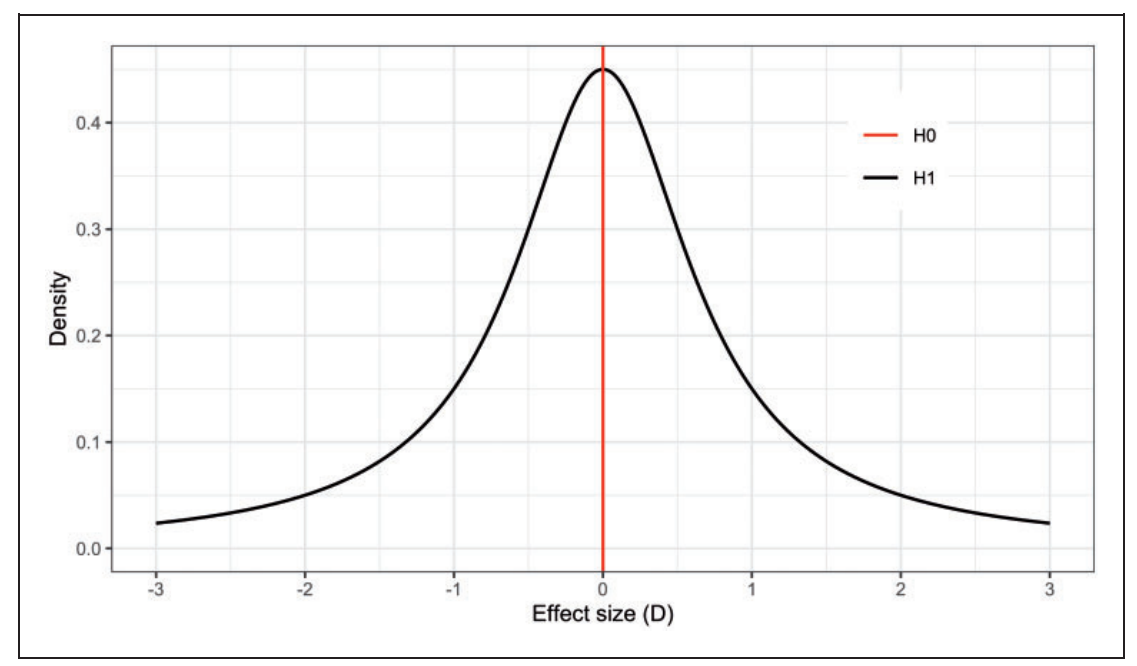

Figure I. Example of two hypotheses compared in BF hypothesis testing. The alternative hypothesis $\left(\mathrm{H}_{\mathrm{I}}\right)$ in black is represented by a two-sided Cauchy distribution centered on zero with an $r$ (width-parameter) of 0.707 . The null hypothesis $\left(H_{0}\right)$ in red, is defined as the point zero value.

from minus infinity to plus infinity, and has fat tails, i.e. it assigns higher probability to more extreme values, relative to other continuous distributions. Using a Cauchy distribution means that the researcher specifies $\mathrm{H}_{1}$ to reflect the belief that the effect (such as a patientcontrol difference) is of small or medium size with relatively high confidence, but also allowing, with less confidence, an effect of a larger size. The fatness of the tails is determined by the width parameter $r$ (analogous to the SD of a normal distribution), and is by convention often set to 0.707 . Formally, the two competing hypotheses in such a BF test are

$$
\begin{gathered}
H_{0}: \delta=0 \\
H_{1}: \delta \sim \operatorname{Cauchy}(0,0.707)
\end{gathered}
$$

where $\delta$ denotes the true population effect, and " $~$ " means "distributed according to." This particular null and alternative-hypothesis pair have become so common today when testing mean differences that it is called the "default" BF t-test, ${ }^{21}$ which can be calculated from a conventional $t$-statistic ${ }^{22}$ (see Supplementary material for an analytical expression of the test). In this tutorial, we will only evaluate sequential testing in PET studies using the default BF t-test.

Example I. We examined cerebral difference in $\left[{ }^{11} \mathrm{C}\right]$ DASB binding potential (an index of serotonin transporter availability) between patients with seasonal affective disorder and healthy control subjects in the winter ( $\mathrm{N}=17$ vs. 23$)$ and summer seasons $(\mathrm{N}=20$ vs. 23) by extracting data from Figure 1 in a study by
McMahon et al. ${ }^{23}$ The group means were compared using a two-sided default BF t-test. In summer, there was 3 times more support in favour of $\mathrm{H}_{0}$ compared to $\mathrm{H}_{1}(\mathrm{BF}=0.32)$. In winter, there was instead 3 times more support in favour of $\mathrm{H}_{1}$ compared to $\mathrm{H}_{0}$ $(\mathrm{BF}=3.01)$, with patients showing higher binding. We can hence conclude that there is moderate evidence of no difference in serotonin transporter availability between patients and controls in the summer season, contrary to the winter season.

Sequential BF testing. In sequential BF testing, the null hypothesis is assessed in constant competition to the alternative hypothesis. If a $\mathrm{BF}$ is calculated after the collection of each data point, it informs the researcher how the stated hypotheses are gaining or losing support from the data. Because of this, the BF can be used for "online" monitoring of incoming data. The researchers can stop when they reach a pre-set decision threshold, a pre-set maximum sample size (or when they simply run out of money, time or patience). ${ }^{12,13}$

However, it is important to note that BF testing is subject to the same sources of uncertainty as NHST inference, i.e. the data could potentially lend support to the wrong hypothesis. This means that in sequential BF testing, the researchers can end up stopping a study early and reach an incorrect conclusion. In order to plan a PET study when intending to use a sequential $\mathrm{BF}$ design, the researchers should be aware of the different errors that can occur when making stopping decisions. False positive evidence is defined as data supporting the alternative hypothesis, when the population effect truly is zero (cf. the type I error in the classical NHST framework). False negative evidence 
is defined as data supporting the null hypothesis, when in fact there is a real effect in the population. It is also possible that the sequential $\mathrm{BF}$ testing procedure ends up unable to stop for either hypotheses, by never passing the decision threshold before a maximum possible fixed sample size (Nmax) is reached. In this tutorial, we refer to such results as an "inconclusive" stopping outcome. Figure 2 depicts the continuous evidence updating, after each added data point, and shows the three possible outcomes: a stop decision for the alternative hypothesis, a stop decision for null hypothesis and an inconclusive stop outcome, when applying sequential BF testing.

If a researcher continues to collect data indefinitely, the default BF t-test will eventually converge to supporting the hypothesis most compatible with the population effect, i.e. it will show evidence for the null-hypothesis if in fact there is no true mean difference, or for the alternative-hypothesis if there is an underlying difference in the population. ${ }^{22}$ However, such continuous collection of data is often unrealistic in a clinical PET study. Apart from the high cost, a PET examination also entails injecting subjects with radioactivity. Hence, a maximum sample size usually has to be decided on a priori and approved by an ethical review board and/or a radiation safety committee.

In summary, $\mathrm{BF}$ is a versatile metric that can be used for different purposes. In the PET literature, there have so far only been a few articles that have used $\mathrm{BF}$ to e.g. complement reported p-values, as a stand-alone metric to quantify the evidence of stated hypotheses, or to assess the replicability of previously published results. ${ }^{24-26}$ Of particular interest for this tutorial, the BF first lends itself naturally to sequential testing of data ${ }^{12,13}$; and second, it quantifies evidence in favour of either $\mathrm{H}_{1}$ and $\mathrm{H}_{0}$, meaning that the nullhypothesis also can gain, or lose, support by the data and hence be formally accepted or rejected. ${ }^{14,22}$ However, as with all metrics used for statistical inference, the BF is not without limitations and criticism. $^{27,28}$ Specifically, when planning to use a sequential testing procedure in a study, it is important to consider the rate of false evidence, as interpreting a $\mathrm{BF}$ outcome based only on evidential thresholds might lead to unacceptable high levels of inappropriate conclusions. ${ }^{29,30}$

In this tutorial we will explore how to use the default $\mathrm{BF}$ t-test to sequentially test data in two common clinical PET study designs: a cross-sectional (e.g. a patient-control comparison) and a paired (e.g. a preintervention-post scan) design. Our first aim is to demonstrate the relative merit of using sequential $\mathrm{BF}$ testing to stop data collection at an earlier stage, compared to simply applying a conventional "fixed N" design, while still keeping the number of false positives below the commonly set threshold of $5 \%$. Our second aim is to assess whether sequential BF testing can be used to stop early, not only when there is an effect in the population $\left(\mathrm{H}_{1}\right.$ is true), but also when there is no effect in the population $\left(\mathrm{H}_{0}\right.$ is true). Our third aim is to

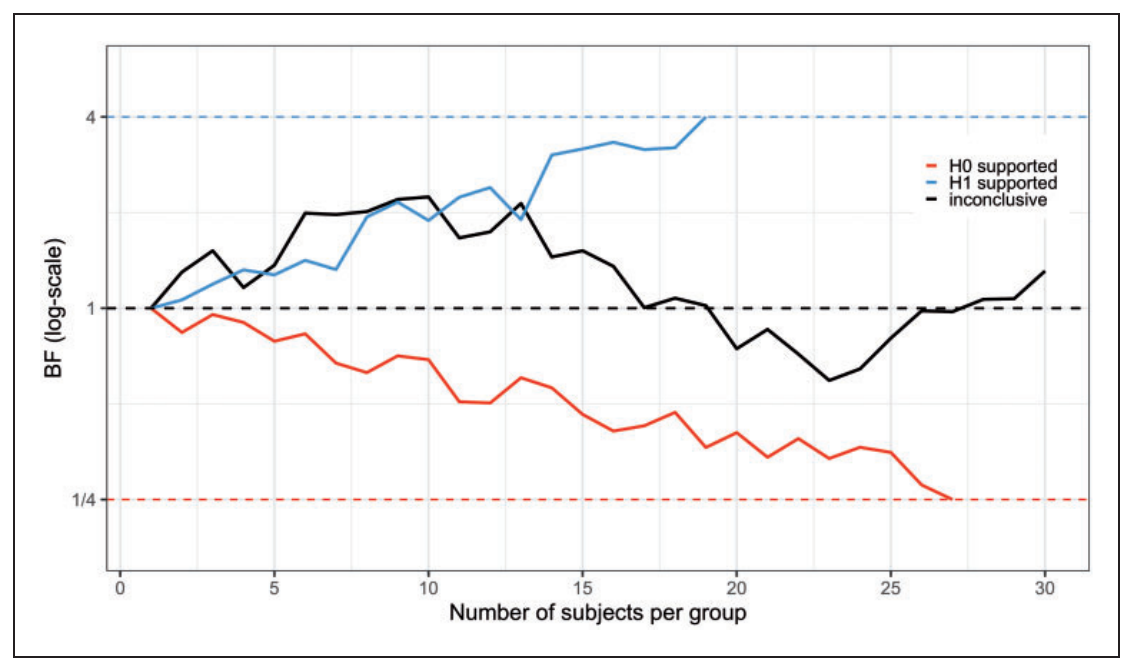

Figure 2. Possible outcomes of sequential testing using Bayes Factor. Three different studies have been initiated, all having $\mathrm{N}=30$ as the maximum possible sample size. For the blue line, BF crosses the pre- specified threshold (here BF $>4$ ) triggering an early stop decision in favor of an effect. Assuming there is a true effect in the population, this would be a "true positive" finding. If there is no effect in the population, this would be a "false positive" finding. For the red line, the reciprocal of BF crosses the pre-specified threshold (here $<\mathrm{I} / 4$ ), meaning that the study is stopped in favor of the null hypothesis. Assuming there is no effect in the population this would be a "true negative", but if there is an effect, this would be a "false negative" finding. For the black line, BF does not reach either below I/4 or above 4 before the pre-specified number of subjects have been reached, and therefore denotes an inconclusive stopping outcome. 
apply sequential BF testing on real clinical PET data, and evaluate the outcome.

Standardized effect sizes. Throughout this tutorial we use standardized effect sizes instead of raw values of e.g. binding potential $\left(\mathrm{BP}_{\mathrm{ND}}\right)$, total distribution volume $\left(V_{\mathrm{T}}\right)$ or percentage differences when we simulate mean differences. To derive a standardized effect for a comparison of two different groups, such as patients and controls, we divide the average difference in the raw outcome by the pooled standard deviation of the two groups, and call the result Cohen's D. ${ }^{31}$ For a comparison within the same individual over time, such as a pre-scan-intervention-post-scan design, we divide the average difference in the raw outcome by the within-subject standard deviation (i.e. the standard deviation of the difference score), and call the result Cohen's Dz. ${ }^{32}$

The main rationale for using standardized effect sizes is that it allows us to generalize the results of the simulations to all radioligands. In Table 2 we present a subset of commonly used radioligands in PET research, ${ }^{33-36}$ and how differences in $\mathrm{BP}_{\mathrm{ND}}$ or $\mathrm{V}_{\mathrm{T}}$ translate into standardized effect sizes and vice versa.

\section{Objective I - Keeping false positive rate below 5\%}

Simulation set-up. Our first aim of this tutorial is to compare sequential BF testing to the conventional way of evaluating research hypotheses in a clinical PET study, which is to apply a single test after the entire sample size has been collected (i.e. a "fixed $\mathrm{N}$ approach").
Here we will explore the differences between sequential $\mathrm{BF}$ testing and taking a fixed $\mathrm{N}$ approach, presenting the reader with the trade-offs that are made when using a sequential procedure. We will show how sequential BF testing can be used in order to stop a study early while still keeping the rate of false positive findings under the commonly set threshold of $5 \%$. In order to compare the sequential BF framework to the conventional approach, we will only stop data collection when there is evidence in favour of an effect. In this simulation, evidence for the null hypothesis will hence be treated the same as inconclusive stopping outcomes, i.e. no early stopping decisions will be made when $\mathrm{BF}<1$. For a demonstration on how to stop a study early also when $\mathrm{H}_{0}$ is supported, see Objective 2 below.

Due to the high cost of performing a PET scan, it is rare to see PET studies with large sample sizes. In order to reflect this reality, our simulations focus on typical cases in PET research, when the study includes $<100$ subjects/group.

Sequential BF testing has been shown to produce errors, leading to wrong decisions, most often when sample sizes are very low. ${ }^{13}$ If the first BF is calculated soon after the initiation of the study (e.g. at $n=3$ subjects/group), then the false positive rate can become unacceptably high. It is therefore sensible to first collect a fixed number of subjects from each group before initiating sequential testing with BF. Below we present the results from simulations within which sequential $\mathrm{BF}$ testing began after data from 12 subjects/group had been collected.

Table 2. Translating standardized effect sizes into raw BPND and VT values for four radioligands.

\begin{tabular}{|c|c|c|c|c|}
\hline Effect size & $\begin{array}{l}{ }^{{ }^{\prime} \mathrm{C} C} \text { ]raclopride } \\
\text { (Putamen) } \mathrm{BP}_{\mathrm{ND}} \\
\text { (\%) difference }\end{array}$ & $\begin{array}{l}\text { [ ' C]AZI04I9369 } \\
\text { (Occipital cortex) } \\
\mathrm{BP}_{\mathrm{ND}}(\%) \text { difference }\end{array}$ & $\begin{array}{l}{\left[{ }^{1} \mathrm{C} C\right] \text { PBR28 }} \\
\text { (Grey matter) } \\
\mathrm{V}_{\mathrm{T}}(\%) \text { difference }\end{array}$ & $\begin{array}{l}{\left[{ }^{\prime \prime} C\right] \cup C B-J} \\
\text { (Putamen) } \\
V_{T}(\%) \text { difference }\end{array}$ \\
\hline \multicolumn{5}{|l|}{ Cohen's D } \\
\hline 0.2 & $0.07(2 \%)$ & $0.03(2 \%)$ & $0.37(10 \%)$ & $0.36(2 \%)$ \\
\hline 0.5 & $0.17(5 \%)$ & $0.07(4 \%)$ & $0.92(26 \%)$ & $0.90(4 \%)$ \\
\hline 0.8 & $0.27(7 \%)$ & $0.12(7 \%)$ & I. 48 (42\%) & $1.43(6 \%)$ \\
\hline 1.2 & 0.41 (I I\%) & $0.18(10 \%)$ & $2.22(63 \%)$ & $2.15(10 \%)$ \\
\hline 1.5 & $0.5 \mathrm{I}(14 \%)$ & $0.22(13 \%)$ & 2.77 (79\%) & $2.69(12 \%)$ \\
\hline \multicolumn{5}{|l|}{ Cohen's Dz } \\
\hline 0.2 & $0.03(1 \%)$ & $0.03(2 \%)$ & $0.12(3 \%)$ & $0.21(1 \%)$ \\
\hline 0.5 & $0.08(2 \%)$ & $0.07(4 \%)$ & 0.30 (8\%) & $0.53(2 \%)$ \\
\hline 0.8 & $0.13(4 \%)$ & $0.11(6 \%)$ & 0.48 (I3\%) & $0.85(4 \%)$ \\
\hline 1.2 & $0.19(5 \%)$ & $0.16(10 \%)$ & $0.71(20 \%)$ & $1.28(6 \%)$ \\
\hline 1.5 & $0.24(7 \%)$ & $0.20(12 \%)$ & $0.89(25 \%)$ & $1.60(7 \%)$ \\
\hline
\end{tabular}

The standardized effect sizes are used to assess the difference between two groups (Cohen's $D$ for a cross-sectional design) and difference within the same subjects (Cohen's $D z$ for a paired design) and have here been translated into raw mean difference in $B P_{N D}$ and $V_{T}$ and percentage mean difference (\%) for four PET radioligands. The mean, SD between subjects and SD within subjects for a representative brain region for each radioligand were taken from test-retest studies on healthy subjects. NB: the variance is likely to be higher in a more heterogeneous clinical population, which will lead to smaller effect sizes for the same raw or \% difference. 
Specifically, for the cross-sectional design we:

1. Simulated a population of patients and control subjects with a true difference between the groups corresponding to $\delta$. We then sampled eleven patients and eleven controls from this population.

2. We sampled one additional patient and one additional control and compared the difference between group means, using the default two-sample BF t-test from the R-package BayesFactor. ${ }^{37}$

3. The data collection was stopped if the BF reached a predefined threshold.

4. If BF did not reach a predefined stopping threshold, we repeated steps 2-3 until we reached Nmax subjects/group. At that point, the data collection was stopped, regardless of the $\mathrm{BF}$ result.

Step 1-4 were then repeated 30,000 times, and the results from the sequential $\mathrm{BF}$ testing were saved. We examined a range of $\delta$ values, from a Cohen's $\mathrm{D}=0$ (no difference between patients and controls) to a Cohen's
$\mathrm{D}=1.5$ (large difference between patients and controls). We also evaluated a range of stopping thresholds, going from 2 (negligible evidence for $\mathrm{H}_{1}$ ) to 10 (strong evidence for $\mathrm{H}_{1}$ ).

The same simulation scheme as above was used for the paired design, with the exception of simulating within-subject differences and applying a paired BF ttest instead of a two-sample test.

Results. Figure 3(a) and (b) show the estimated false positive rate when applying sequential $\mathrm{BF}$ testing in the case in which there is no difference between the two groups. Three different stopping thresholds have been used ( $\mathrm{BF}>3,4$ and 6 , respectively). When the maximum allowed sample size increases, the false positive rate goes up. This is because the longer the sequential testing can go on, the more decisions are being made. Some of these decisions will be wrong, meaning that the researcher will claim support for an effect, when in fact there is no effect in the population. Eventually, when $\mathrm{N}$ becomes high enough, the rate of

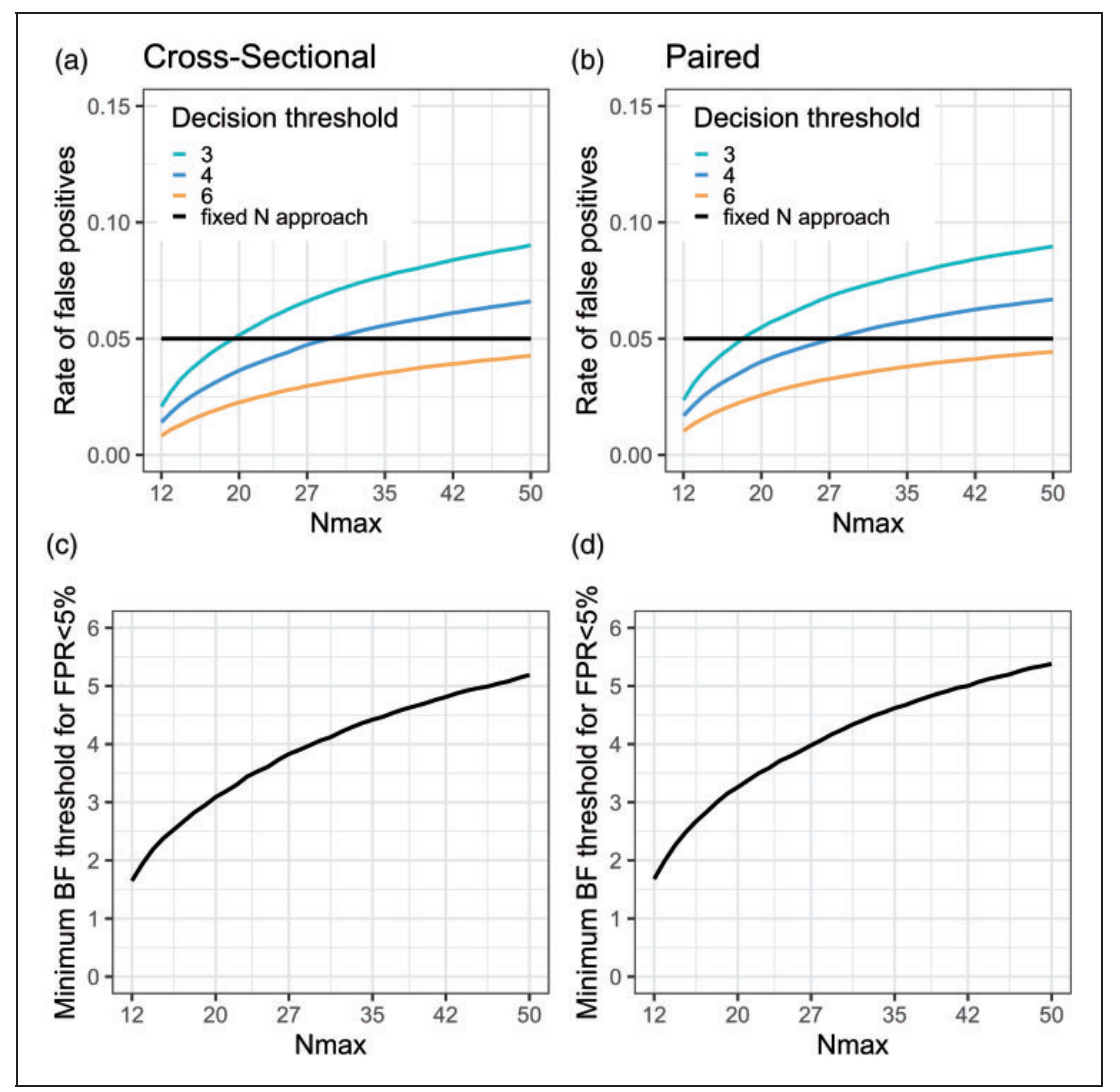

Figure 3. ( $a$ and $b$ ) The rate of false positive stopping decisions increases as the maximal number of subjects per group (Nmax) becomes higher due to an increasing number of decisions being made, shown for three different BF decision thresholds. (c and d) The $\mathrm{BF}$ decision threshold can be adjusted to achieve a desired rate of false positive evidence (here 5\%) for different Nmax. For all figures: samples are drawn from populations showing no (between or within) mean difference; testing starts at $\mathrm{N}=12$ subjects/group; and $\mathrm{BF}$ is checked after every additional comparison pair (I set of patient-control scans or pre-post scans). FPR: false positive rate. 
false positives will reach an asymptote (see Supplementary Figure S1). With the settings chosen for Figure 3(a) and (b), and a maximum sample size above 30 subjects/group, it is not possible to keep false positives below $5 \%$ using a decision threshold of 4 . If the researchers wish to control the false positives at the $5 \%$ level, several options are available: start testing later than $\mathrm{N}=12$, test less often (e.g. after every second or third added patient-control pair), choose a higher $r$ value for the Cauchy distribution, or, increase the decision threshold. Figure 3(c) and (d) show the estimated decision threshold that would be needed to keep the false positive rate at $5 \%$ when using sequential testing with the default BF t-test, while still starting at 12 subjects/group and checking the BF after the collection of each patient-control pair.

Figure 4 shows the results from the simulations when $\mathrm{Nmax}=30$ subjects/group, using a decision threshold just above 4 . In the upper panel the rate of positive studies at different population effects is visualised. The curves cross the $\mathrm{y}$-axis at $\mathrm{D}=0$ (i.e., the false positive rate), $5 \%$ for both sequential $\mathrm{BF}$ testing and the conventional fixed $\mathrm{N}$ test. As can be seen, had the researcher instead applied a fixed $\mathrm{N}$ approach (at 30 subjects/group), they would have increased the power to detect the population effect by no more than $\sim 10 \%$ for any effect size compared to using sequential testing. Note that when calibrating the $\mathrm{BF}$ threshold to correspond to a $5 \%$ false positive rate, and stopping only for $\mathrm{H}_{1}$, the $\mathrm{BF}$ procedure will show identical results to performing sequential NHST t-tests with a $5 \%$ corrected significance level. Hence, this approach will be identical to the blue line in Figure 4.

Figure 4(c) and (d) show the average number of subjects needed to reach a decision at different population effects. As the underlying difference between the two populations is increased, fewer subjects are needed to reach a decision using sequential BF testing. Already at a true population difference of Cohen's $\mathrm{Dz}=0.5$, researchers will on average save $30 \%$ in terms of both expense and amount of injected radioactivity when
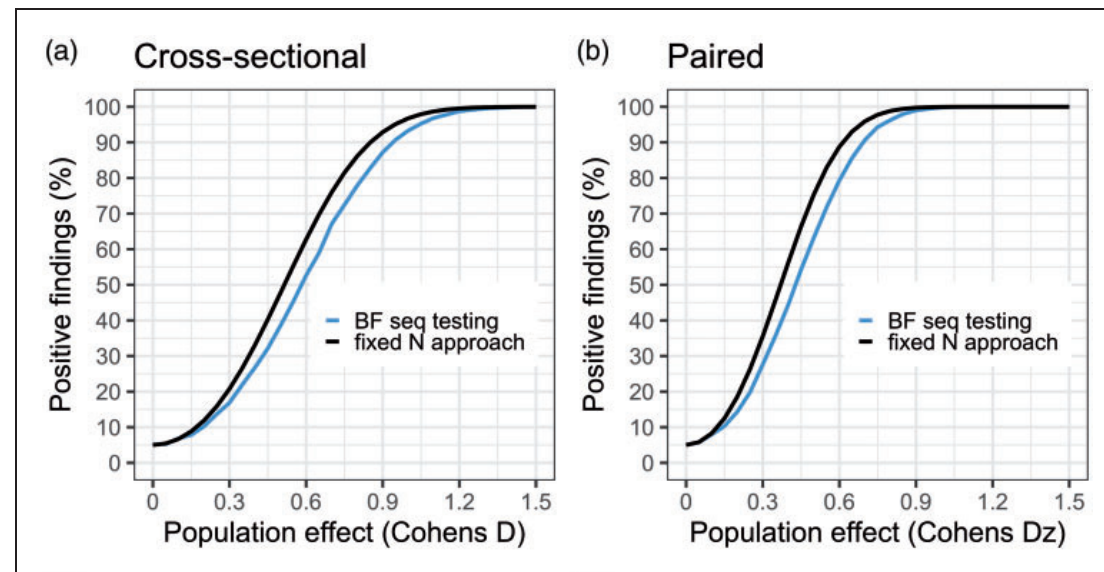

(c)

(d)
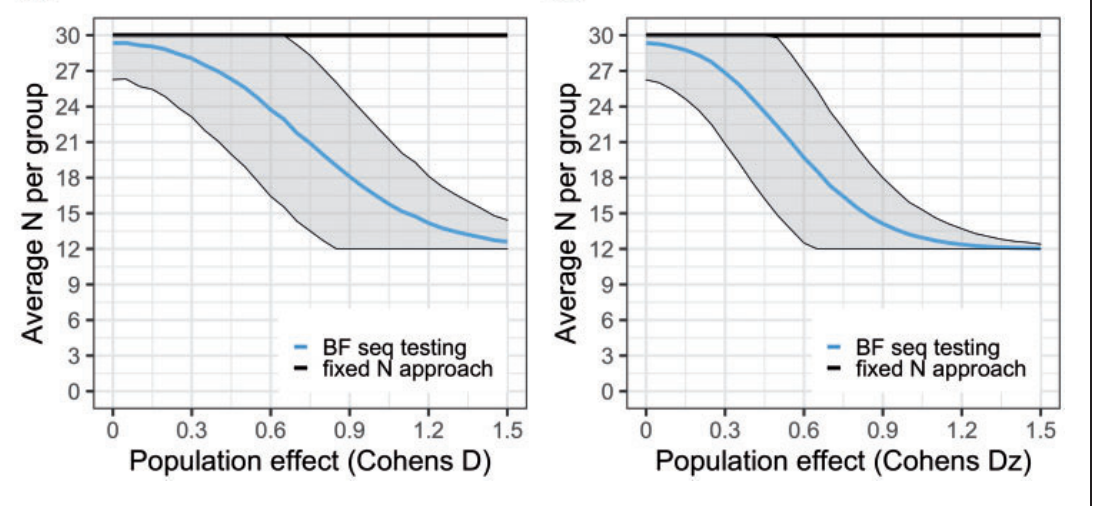

Figure 4. Panels (a) and (b) show the proportion of studies that showed support for HI (aka "power curves") for BF sequential testing (blue) and fixed $\mathrm{N}$ test (black), at different population effects (starting at $\mathrm{D}=0$, i.e. no effect). For the fixed $\mathrm{N}$ approach, only one test is performed at $\mathrm{N}=30$ subjects/group. For the sequential testing, I 2 subjects/group are first collected, then BF is checked after each added comparison pair until 30 subjects/group is reached, stopping the study when BF $>4$. Panels (c) and (d) show the average number of subjects needed to reach a stopping decision at different population effects. The fixed $\mathrm{N}$ test is the black line $(\mathrm{N}=30$ subjects/group); BF sequential testing is the blue line with shaded area denoting \pm ISD. 
applying sequential BF testing compared to the fixed $\mathrm{N}$ approach.

In summary, if the PET researchers apply a sequential testing procedure as presented here, they have the potential of reducing both expense and radioactivity exposure, however, it is important to note that this procedure comes at a cost of a lower rate of true positives (i.e. statistical power), for a range of effect sizes.

Simulation results for different choices of Nmax $(15, \quad 20, \quad 50$ and 100 subjects/group $)$ are presented in Supplementary material (Supplementary Figures S2 to S5).

Practical recommendations $I$. In this simulation set-up we show that it is possible to stop a PET study early if there is a true underlying effect in the population, while keeping the number of false positive findings below $5 \%$. We recommend to not start sequential $\mathrm{BF}$ testing until at least 12 subjects per group have been collected, adjusting the BF threshold upwards for larger values of Nmax. Importantly, these settings should only be used when the PET researcher is interested in stopping early when data shows support for $\mathrm{H}_{1}$. All BF values showing support in favour of $\mathrm{H}_{0}(\mathrm{BF}<1)$ should not, with these settings, be interpreted as anything else but inconclusive stopping outcomes.

\section{Objective 2 - Stopping for both $H_{1}$ and $H_{0}$}

The second aim of this tutorial is to assess whether sequential BF testing can be used to stop a clinical PET study early, both when there is an effect in the population, but also when the population effect is zero. After the collection of each data point, we will compare both the $\mathrm{BF}$, and its reciprocal, to an a priori set decision threshold. If either the $\mathrm{BF}$ or its reciprocal passes the threshold, the study will be stopped, and we will declare support in favour of the alternative or null hypothesis, respectively.

Simulation set-up. We used the same simulation set-up as above, where a grid of maximum sample sizes and effect sizes ranging from 0 to 1.5 was used to evaluate sequential $\mathrm{BF}$ testing in a cross-sectional and paired design respectively. We used the same settings for the default BF t-test as above with one exception: the alternative hypothesis was still a Cauchy distribution (centered at zero with $r=0.707$ ) but now specified as being one-sided, instead of two-sided. This means that we anticipate that the effect will go in one direction (e.g. patients will have a higher $\mathrm{BP}_{\mathrm{ND}}$ than controls), making the test into a one-sided default $\mathrm{BF}$ t-test. The reason for considering only a one-sided test in this scenario is that it is not possible to stop for $\mathrm{H}_{0}$ when using a two-sided test at smaller sample sizes when using reasonable decision thresholds $(\mathrm{BF}<1 / 4$, see Supplementary Figure S6).

In the simulations reported below, a one-sided default BF t-test was therefore performed sequentially, after 12 subjects per group had been collected. If no decision was reached after 30 subjects/group had been collected, the study was stopped and the result was considered to denote an inconclusive stopping outcome. The decision threshold was set to 4 and $1 / 4$, for the alternative and null hypothesis respectively.

Results. Figure 5 summarises the results from the simulations. When the population difference is zero in the cross-sectional design, a study will stop (incorrectly) for $\mathrm{H}_{1}$ just above $5 \%$ of the time, and (correctly) for $\mathrm{H}_{0}$ about $60 \%$ of the time. In this case, studies will be able to stop, on average, after just 21 subjects/group have been scanned. Hence, assuming there is no true difference between groups, a sequential BF testing procedure will on average save 18 PET examinations, i.e. $30 \%$ in terms of expense and exposure, compared to the strategy of collecting data until Nmax is reached.

For the paired design we observe a similar false positive rate but a higher rate of true negative evidence $(\sim 75 \%)$ when the population effect is zero. The rate of true positives is also higher at all evaluated effect sizes compared to the cross-sectional design (Figure 5). For example, at a population effect of $\mathrm{Dz}=0.5$, a stop decision is reached before or at Nmax in $75 \%$ of studies. In this case, studies can on average be stopped after scanning 19 subjects/group, saving in total 22 examinations, i.e. $36 \%$ expense and injected radioactivity.

The area around the point where the red and black line cross, is a weak spot for the BF sequential testing procedure. At this range of true effects in the population, the risk of obtaining an inconclusive result is at its highest (i.e. the blue line is at its nadir). In the cases in which a decision is reached, the study will stop with equal probability for $\mathrm{H}_{1}$ and $\mathrm{H}_{0}$, and the risk for false negative evidence is around $25 \%$.

Assuming that the PET researcher would be interested in stopping a study early when $\mathrm{H}_{0}$ is supported, a range of population effects with high risk of false negative evidence will always exist. It is therefore sensible to beforehand decide on a minimal population effect of interest, and choose the settings to ensure that any larger effect does not have too high a risk of generating evidence for $\mathrm{H}_{0}$. For example, with the settings presented in Figure 5 (Nstart $=12, \mathrm{Nmax}=30$, threshold $=4)$ the risk of false negative evidence is $>10 \%$ for effects between $\mathrm{D}=0$ and 0.45 .

The same results but for different $\operatorname{Nmax}(15,20$, 50 and 100 subjects/group) are presented in Supplementary material (Supplementary Figures S7 to $\mathrm{S} 10)$. 

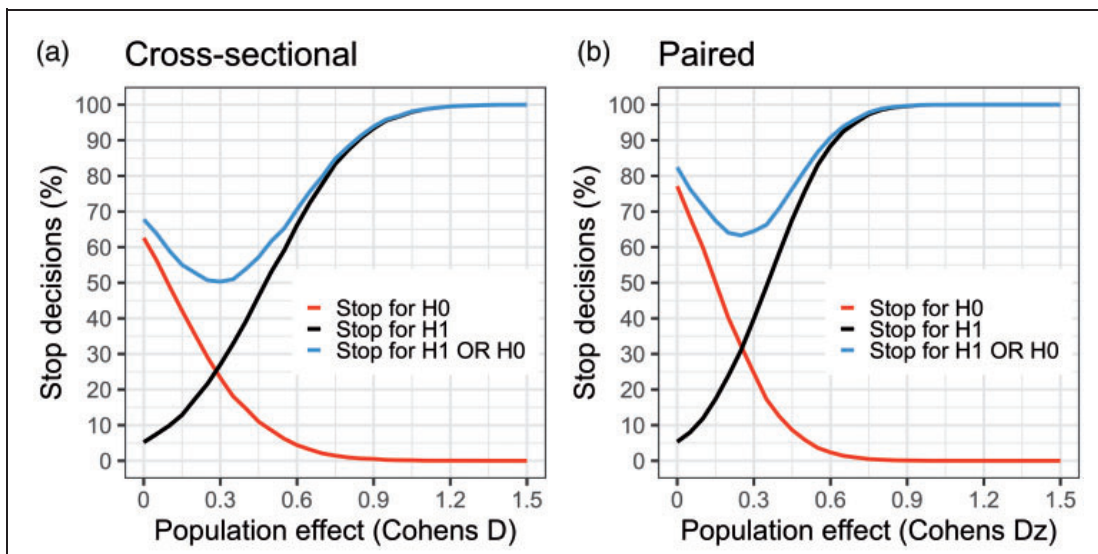

(c)

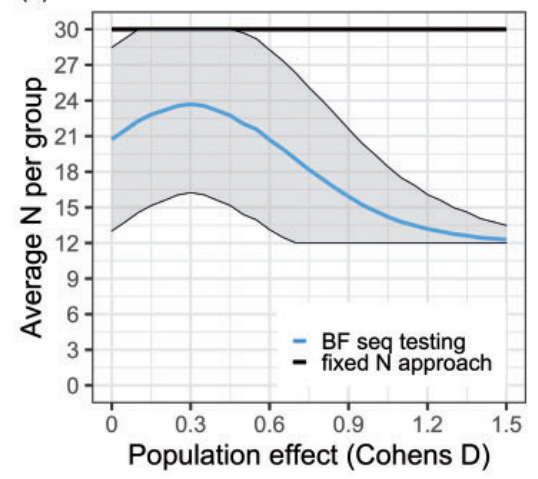

(d)

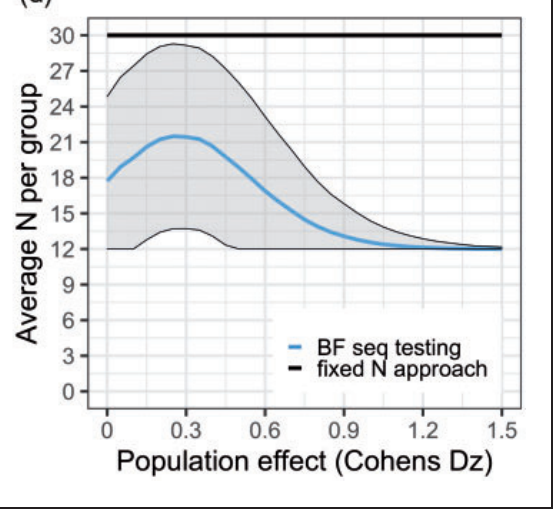

Figure 5. (a) and (b) The black curve denotes the proportion of studies that showed support for HI (BF $>4$ ) during data collection, at a range of population effects (starting at no effect, $D=0$ ). The red curve is the proportion of studies that showed support for $\mathrm{H} 0$ $(\mathrm{BF}<\mathrm{I} / 4)$. The blue curve is the sum of the red and black curves. It describes the proportion of studies that yield a stopping decision, either in favour of $\mathrm{HO}$ or in favour of $\mathrm{HI}$. Since the true population effect is unknown to the researcher, the blue curve can be viewed as the probability of reaching a stopping decision regardless of it being due to true or false evidence. When there is no effect in the population $(D=0)$, the black curve shows the rate of false positives. At this point, the red curve shows the probability of correctly stopping early for $\mathrm{HO}$ (i.e. proportion of true negatives). (c) and (d) shows the average number of subjects needed to reach a stopping decision at different population effects. The flat black line represents $\mathrm{Nmax}$ (30 subjects/group). BF sequential testing is the blue line with shaded area denoting \pm ISD.

Practical recommendations 2. In this simulation set-up we show that it is possible to stop a PET study early either when there is a true underlying effect in the population, or when the effect is zero. When using the Cauchy distribution to describe the alternative hypothesis, we recommend that sequential BF testing does not start until at least 12 subjects per group have been collected, setting the BF threshold to a minimum of 3 , and using a one-sided test. Using a one-sided test means that the researcher must make an a priori prediction of which direction the effect will have, and not change this prediction after the study is started, similar to performing a classical one-sided NHST t-test. Making a one-sided prediction can be desirable in molecular imaging research, as long as it is well anchored in previous literature or theory. This decision should, however, be part of the research question and overall study design, and not simply made to fulfill the criteria for performing a statistical procedure.

\section{Objective 3 - Application to real clinical PET data}

The third and final aim of this tutorial is to apply sequential BF testing to a real clinical PET setting. To this purpose, we used already collected data of patients with major depressive disorder and healthy control subjects examined with $\left[{ }^{11} \mathrm{C}\right] \mathrm{WAY} 100635$, a radioligand which binds to the serotonin $1 \mathrm{~A}$ (5HT1A) receptor. ${ }^{38,39}$ From this data, we included 40 medication-free patients (mean Age 36.2 (12.9 SD); 25 females) and 40 healthy controls (mean Age 37.1 (14.0 SD), 25 females). The 5HT1A receptor acts inhibitory on serotonergic neurons in the raphe nuclei, and a high concentration of receptors in this structure 
will likely lead to lower transmitter release in serotonergic projection areas. Hence, we hypothesized that patients suffering from MDD show higher 5HT1A receptor availability in the raphe nuclei compared to healthy controls. Here we apply sequential BF testing to examine whether this hypothesis gains enough support to stop data collection before 40 subjects per group are reached.

Methods. $\mathrm{BP}_{\mathrm{F}}$, defined as the ratio of concentration of specifically bound radioligand in the brain region to the concentration of free radioligand fraction in arterial plasma at equilibrium, was used as the primary outcome measure for assessing 5HT1A receptor availability in the raphe nuclei. Cerebellar white matter was used as reference tissue.

We applied sequential $\mathrm{BF}$ testing to assess the support in data in favor of patients having higher $\mathrm{BP}_{\mathrm{F}}$ in the raphe nuclei than controls $\left(\mathrm{H}_{1}\right)$, as compared to no difference $\left(\mathrm{H}_{0}\right)$. We also examined the evidence in data in favor of $\mathrm{H}_{0}$ over $\mathrm{H}_{1}$, to stop the study early if the null hypothesis was supported. The stopping threshold was set to $\mathrm{BF}>5$ for $\mathrm{H}_{1}(\mathrm{BF}<1 / 5$ for $\mathrm{H}_{0}$ ), in order to keep the rate of false positive evidence below 5\% (see Supplementary Figure S1). We used a one-sided Cauchy distribution, centered around 0 with an $r=0.707$ to describe the predicted mean difference under $\mathrm{H}_{1} \cdot \mathrm{H}_{0}$ was specified as the point zero value.

First, patient and control data were sorted according to the chronological order in which the subjects were examined. We then retrieved the first 12 patients and 12 control subjects, standardized all raphe nuclei
$\mathrm{BP}_{\mathrm{F}}$ values, calculated the $\mathrm{BF}$ and compared it against the stopping thresholds. Following this, we added one additional patient and one healthy control to the previous subjects, checked $\mathrm{BF}$ against the thresholds, and so on, until we either were able to stop early, or reached the maximum of 40 subjects/group.

Results. When applying a one-sided two-sample t-test to the full dataset, we observed a large group difference in raphe nuclei $\mathrm{BP}_{\mathrm{F}}(\mathrm{t}=4.1, \mathrm{df}=76.58$, $\mathrm{p}=0.00006, \mathrm{BF}=385$ ), with patients showing higher values compared to healthy controls $(\mathrm{D}=0.91$ or a $51 \%$ increase).

When applying sequential testing, the BF passes the threshold of 5 in support of $\mathrm{H}_{1}$, after the inclusion of 27 subjects/group (Figure 6). Hence, had sequential testing been applied in collection of $\left[{ }^{11} \mathrm{C}\right] \mathrm{WAY} 100635$ patient-control data, it would have been possible to stop the recruitment at a total $\mathrm{N}$ of 54 instead of 80 , saving $33 \%$ in terms of expense and radioactivity exposure. Assuming that a PET examination costs 5000 USD/Euro, that would amount to saving 130,000 USD/Euro in total.

When using the same settings as described above, but permuting the order in which patients and controls were recruited to this study, a stop decision could be taken on average at 17 subjects/group $(\mathrm{SD}=5.9$, range 12-33). We also included a comparison between BF sequential testing and two common NHST alpha spending approaches, which showed very similar stopping decisions for this dataset (see Supplementary Figure 11).

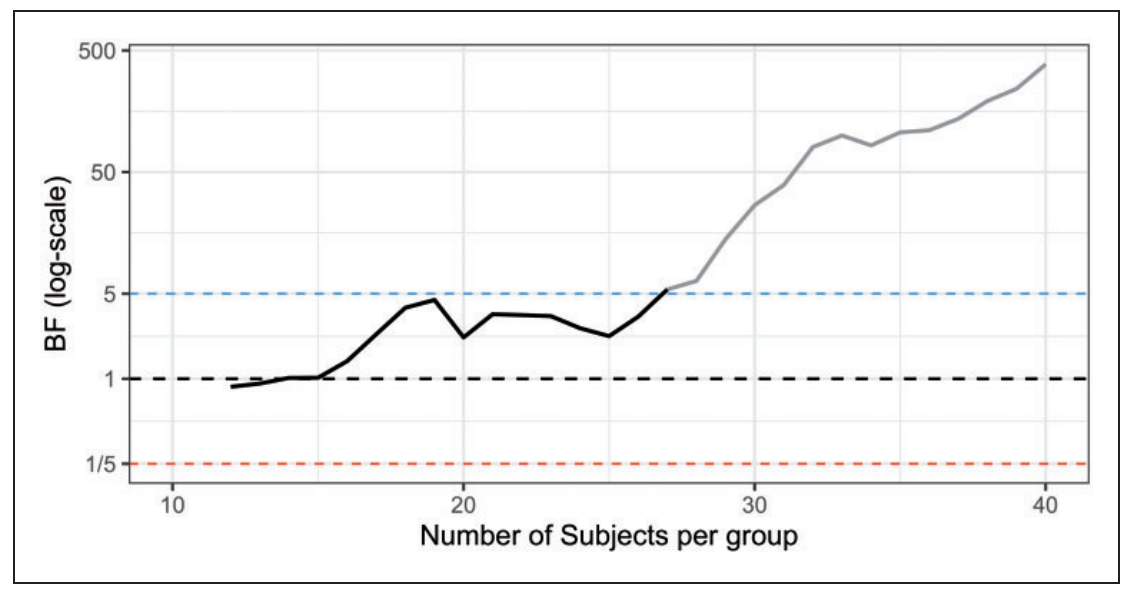

Figure 6. Sequential BF testing applied to real PET data. Patients with major depressive disorder were compared to healthy control subjects using [ ${ }^{\prime} \mathrm{C}$ ]WAY $100635 \mathrm{BPF}$ as a measure of 5 HTIA receptor availability in the raphe nuclei. BF testing starts after 12 subjects/group have been collected and a stop decision is triggered at 27 subjects/group. The blue line denotes the stopping threshold for $\mathrm{HI}$ (set to 5) and the red line the stopping threshold for $\mathrm{HO}$ (set to I/5). The black line shows the change in BF following the inclusion of each additional patient-control pair. The grey line shows how the BF trajectory would have looked, assuming no stopping decision was made, and the data collection instead continued up until the full sample of 40 subjects/group. 


\section{Discussion}

In this tutorial we show that it is possible to stop recruitment of subjects in PET studies as soon as enough data have been collected to make a conclusion, reducing both expense and exposure to radioactivity. We do this by employing sequential $\mathrm{BF}$ testing, which assesses the support in data in favor of two competing hypotheses while the study is still ongoing.

When applying sequential BF testing, and the true population effect is small, the savings are modest compared to using the conventional design, where only one test is performed at a fixed sample size. As the population effect becomes bigger however, it is possible to (on average) stop the data collection early potentially saving more than half of the resources that would be needed for a PET study carried out with the conventional approach (e.g. in Figure 5(d), the average sample size needed is $<15 /$ group for population effects larger than $\mathrm{Dz}=0.7$, compared with fixed $\mathrm{N}$ of 30 /group). For a range of effects, a fixed $\mathrm{N}$ test will however show around $5-10 \%$ higher true positive rate compared to sequential BF testing (Figure 4(a) and (b)). By using a lower BF decision threshold, the proportion of true positive stopping decisions will increase when there is an effect, but it comes with a cost of more false positives when $\mathrm{H}_{0}$ is true.

\section{Alternative sequential procedures}

There are sequential testing methods other than $\mathrm{BF}$ that can be used to stop the data collection early, and that offer exact control over error rates. For the NHST framework, as mentioned in the introduction, a viable alternative is to apply a so-called group-sequential design were the significance threshold is corrected so that the total rate of false positive does not surpass a pre-set alpha value. ${ }^{6}$ In fact, when calibrating the BF threshold (using a "default" Cauchy-distribution for describing $\mathrm{H}_{1}$ ) to a $5 \%$ false positive rate when stopping only for $\mathrm{H}_{1}$ (see Objective 1), the sequential $\mathrm{BF}$ procedure will be close to identical to performing corrected sequential NHST t-tests using so called Pocock boundries. For NHST, there are several different flexible strategies for doing sequential testing to stop a study as soon as $\mathrm{H}_{0}$ can be rejected. ${ }^{40-42}$ There are also methods for performing so called "futility testing," with which the researchers can stop a study early if it is deemed to unlikely that a significant finding will be achieved later on. ${ }^{43}$ Addressing such sequential-NHST procedures for application in clinical PET research are however outside the scope of this tutorial.

\section{Evidence in favor of $\mathrm{HO}$}

Sequential BF testing allows a PET study to be stopped also when there is evidence in favor of no effect in the population, i.e., when $\mathrm{H}_{0}$ is true. If the researcher wants to use a sequential $\mathrm{BF}$ to assess support for both $\mathrm{H}_{1}$ and $\mathrm{H}_{0}$, we recommend using a one-tailed default $\mathrm{BF}$ t-test. Without using a one-sided prediction, it will be difficult (or even impossible) to stop a study early in support for $\mathrm{H}_{0}$, given the sample sizes used in typical PET studies $(\mathrm{N}<100$ subjects/group) while still using a threshold that is high enough to keep the rate of false evidence at reasonable levels (see Supplementary Figure S6).

When allowing a study to be stopped early for $\mathrm{H}_{0}$, false negative evidence must be considered as an additional type of error, and PET researchers now have to consider keeping both false positive and negative evidence low. To do so, the researcher has three main options to their disposal:

1. Increase the a priori set decision threshold for stopping (see Figure 3).

2. Recruit and scan a larger pool of subjects $(>12 /$ group) before initiating the sequential BF testing.

3. Check BF less frequently, e.g. only after the collection of each second or third (etc.) comparison pair, instead of after each single one.

These three approaches could be used separately or in combination with each other. They do all however trade off against a higher number of included subjects, meaning that the study will, on average, require more subjects before a stop decision can be made.

Figure 5 presents the true and false evidence rates the researchers can expect when using a one-sided BF ttest and when they are correct in their prediction of the direction of an effect. If there is a sizable patientcontrol difference in the population, but it goes in the opposite direction to the predicted one, then using this approach would lead studies to be stopped early due to the null-hypothesis being supported almost $100 \%$ of the time. Of course, the null is not true in this case, but it is closer to the true population effect than the a priori defined alternative hypothesis. Hence, it will often gain the most support from the data.

When allowing stopping for $\mathrm{H}_{0}$ as well, the researcher should be aware that the false negative evidence rate can become large when the effect is close to zero, but not exactly zero. Using sequential BF testing, researchers will run the risk of stopping the study, claiming support for no effect, when there is in fact a true but small effect in the population. Whether or not this is acceptable to the researchers depends on how small an effect has to be, in order for the researcher to consider 
it practically indistinguishable from zero. Using the sequential $\mathrm{BF}$ approach with the settings described in Figure 5 (e.g. a decision threshold of 4 ), the false negative evidence rate becomes e.g. $>10 \%$ for all population effects between 0 and 0.45 , and $>5 \%$ for all effects between 0 and 0.6 for a cross-sectional design. Before applying a sequential BF testing procedure where stop for $\mathrm{H}_{0}$ is allowed, PET researchers should therefore consider what range of effects they consider as practically or clinically relevant, and what rate of false negative evidence they are prepared to allow for effects within this range (see Example 2 below).

However, many PET studies are exploratory in nature, and it can be difficult to decide a priori what effect size constitutes a biologically meaningful difference. Here, it can be helpful to think about the effect size specified in a conventional power-analysis required in most clinical PET studies, which ought to be informed by the minimal effect of interest, or in some cases, by Nmax restrictions.

Example 2. There is a true difference in $\left[{ }^{11} \mathrm{C}\right]$ raclopride BPND between a patient and a control population that is of size Cohen's $\mathrm{D}=0.2$ (a difference of 0.07 BPND or $2 \%$, Table 2). For such a small effect, studies will often be stopped because $\mathrm{H} 0$ is supported when applying the sequential $\mathrm{BF}$ testing procedure, e.g. when using a threshold of 4 and $1 / 4$, respectively. A PET researcher might consider such an effect to be practically indistinguishable from zero. In such a scenario, support for $\mathrm{H}_{0}$ is hence of little concern, or even a preferred outcome for the researcher. On the other hand, if the researcher considers a Cohen's $\mathrm{D}=0.2$ to be clinically relevant, then a sequential $\mathrm{BF}$ procedure using the default $\mathrm{BF}$ t-test cannot reliably give the correct stopping decision for $\mathrm{H}_{1}$ in commonly seen sample sizes in PET research $(<100$ subjects/group).

If the researcher wants to detect small effects, they need to use a different specification of $\mathrm{H}_{1}$, or a higher decision threshold (e.g. $\mathrm{BF}>10$ ) together with a much larger Nmax.

General considerations. For the reader who is new to the $\mathrm{BF}$, it might look peculiar to select an alternative hypothesis that not only includes $\mathrm{H}_{0}$ (point zero) but also places the highest probability mass at that point (see Figure 1). The alternative hypothesis in this case ought however to be interpreted as expressing the predicted plausibility of observing an effect in a range of values, rather than single points. This Cauchy distribution says that the researcher, before seeing the data, finds values in the range just around zero to be more likely than values far away from zero.

Throughout this article we have used a Cauchy distribution with an $r$ of 0.707 to describe the alternative hypothesis. However, for any given study design a more appropriate choice of $\mathrm{H}_{1}$ might exist. If the researchers are interested in detecting smaller effects in the population, they can specify $\mathrm{H} 1$ to assign larger plausibility to smaller differences, by lowering the width of the Cauchy (setting $r$ to, e.g. 0.5 ) when planning the study. The latter will increase the rate of true positive evidence at smaller effects in the population, but it will also increase the rate of false positive evidence when $\mathrm{H} 0$ is true.

If a distribution with a higher $r$ (e.g. 1) is used to define $\mathrm{H}_{1}$, then the researcher assigns more predictive weight to a mean-difference further away from 0 . Doing so makes it easier to obtain evidence in favor of $\mathrm{H}_{0}$ (when there is no effect), but harder to obtain evidence for $\mathrm{H}_{1}$ when the true effect is small (see Supplementary Figure S12). This means that in using a larger $r$ than 0.707 (all other settings the same); (1) the rate of false positive evidence will be lower; (2) the researcher will on average stop earlier when $\mathrm{H}_{0}$ is true; (3) small but true effects will more often produce false negative evidence (but see Example 2).

In addition to the settings presented in this tutorial, there are several modifications that can be made for the $\mathrm{BF}$ test. For example, different decision thresholds can be applied for $\mathrm{H}_{1}$ and $\mathrm{H}_{0}$ depending on whether the researcher thinks that false positives are more important to avoid than false negatives, or vice versa.

This tutorial assumes that, for a cross-sectional design, a scanned patient is always followed by a scanned control subject. In a real PET study, this is not always a feasible recruitment scheme. The results above also assume that PET researchers base their stopping decision on the outcome from one primary region of interest. If a researcher applied sequential $\mathrm{BF}$ testing, but cherry-picks the outcome from two or more regions to make a stopping decision, the risk for obtaining false evidence will increase. Finally, this tutorial only applies to study designs where a twosample or paired t-test are suitable, and the reader cannot assume that the results would be similar if e.g. a regression model with covariates were to be used instead.

If PET researchers wish to use settings not discussed in depth in this tutorial: e.g. custom specification of $\mathrm{H}_{1}$, different decision thresholds for $\mathrm{H}_{1}$ and $\mathrm{H}_{0}$, other recruitment schemes, and/or statistical models, we recommend that they modify our simulation code to evaluate their own study design before starting the data collection. All code can be found freely available on https://github.com/pontusps/Early_stopping_in_PET.

Practical recommendation 3. If the researcher has an informed idea on how the studied effect will look, they should consider using specification of $\mathrm{H}_{1}$ other 
than the "default" zero-centered Cauchy employed in this tutorial. This could e.g. be a normal distribution centered around a value considered to be either a plausible group difference or is clinically meaningful. This recommendation also applies to the null-hypothesis; we encourage the researcher to consider redefining $\mathrm{H}_{0}$ in order to test (what might be) a more appropriate hypothesis. An example of this could be to assess if an increase in patient $\mathrm{BP}_{\mathrm{ND}}$ gains more support in data compared to a decrease, rather than just testing "there is a difference" vs. "there is no difference".

Inconclusive stopping outcomes. For any given PET-study where sequential BF testing is applied there will be a chance of reaching Nmax without crossing the pre-set decision threshold. In such cases, where the researcher ends up with an "inconclusive" stop decision, the BF is still interpretable. The suggested evidence thresholds in Table 1 can be used to report the support in data in favor of the two competing hypotheses, even though $\mathrm{BF}$ never formally reached the a priori set stopping threshold. For example, if the $\mathrm{BF}$ is above 3 (or below $1 / 3$ ), this can still be reported as "moderate" support in data for one hypothesis over the other. However, in doing so the researcher cannot any longer say that they are controlling the rate of false evidence at a prespecified level.

Specific recommendations. Before applying the sequential BF testing method, the researchers should decide what they want to prioritize: keeping the risk of making the wrong stopping decision low but accepting more inconclusive stopping outcomes, or stopping as early as possible but with a higher risk of errors. To help decide on this trade-off when planning PET studies, we have developed $\mathrm{R}$ functions with which PET researchers can examine how different settings for the sequential testing approach affects the average sample size needed, as well as rates of true and false evidence (https://github. com/pontusps/Early_stopping_in_PET).

It should by now be clear to the reader that a set of critical decisions needs to be made before applying sequential BF testing in a clinical PET study. For this reason, we recommend all researchers to pre-register their analysis before data collection starts. ${ }^{44-46} \mathrm{~A}$ preregistration can be thought of as a safety net for the researcher. It helps guide the analysis and interpretation of data so that error rates are kept under control. It can also be shown to reviewers or readers to increase the credibility of the methods and findings. Deviations from a pre-registration is of course possible, and often warranted, but should be reported transparently in the article. See e.g. https://aspredicted.org/ for easy and efficient pre-registration.
If a PET researcher wants to perform a study using sequential BF testing, we recommend that they follow the steps outlined in a flow-chart found in Supplementary material (Supplementary Figure S13). In order to perform the default BF t-test, the freely available BayesFactor package ${ }^{37}$ in $\mathrm{R}$ or point-andclick software JASP $^{47-49}$ can be used.

Caveats. While a sequential testing procedure often allows the researcher to stop a study early, an important caveat is that the estimated effect size can become upwards biased. If a study can be stopped early due to $\mathrm{H}_{1}$ being supported, it is more likely that the observed effect size is above, rather than below, the true population effect. ${ }^{13,40}$ This caveat should be considered before interpreting the effect size from a PET study that was stopped early, or before entering such an effect size into a meta-analysis.

Further reading. If the reader is interested in learning more about sequential testing the following articles are a good start for a BF approach: ${ }^{11-13}$ and for a NHST approach. ${ }^{40-42}$

\section{Acknowledgements}

We would like to thank Granville J. Matheson for valuable feedback on this project.

\section{Funding}

The author(s) disclosed receipt of the following financial support for the research, authorship, and/or publication of this article: This work was supported by the Lundbeck Foundation and the Swedish Society for Medical Research.

\section{Declaration of conflicting interests}

The author(s) declared no potential conflicts of interest with respect to the research, authorship, and/or publication of this article.

\section{Supplemental material}

Supplemental material for this article is available online.

\section{Data and code availability}

The data code for reproducing the results, tables and figures in this article can be found at https://github.com/pontusps/ Early_stopping_in_PET.

\section{ORCID iDs}

Jonas E Svensson (D) https://orcid.org/0000-0002-2331-4913 Pontus Plavén-Sigray (D) https://orcid.org/0000-0001-53425641 


\section{References}

1. Van Der Aart J, Hallett WA, Rabiner EA, et al. Radiation dose estimates for carbon-11-labelled PET tracers. Nucl Med Biol 2012; 39: 305-314.

2. Perezgonzalez JD. Fisher, Neyman-Pearson or NHST? A tutorial for teaching data testing. Front Psychol 2015; 6: 223.

3. Strube MJ. SNOOP: a program for demonstrating the consequences of premature and repeated null hypothesis testing. Behav Res Methods 2006; 38: 24-27.

4. Albers C. The problem with unadjusted multiple and sequential statistical testing. Nat Commun 2019; 10 : 1921.

5. Pocock SJ. Group sequential methods in the design and analysis of clinical trials. Biometrika 1977; 64: 191-199.

6. Demets DL and Lan KKG. Interim analysis: the alpha spending function approach. Stat Med 1994; 13: 1341-1352.

7. Selwyn MR and Fish SM. Choice of alpha spending function and time points in clinical trials with one or two interim analyses. Pharm Stat J Appl Stat Pharm Ind 2004; 3: 193-203.

8. Jeffreys H. Theory of probability. 3rd ed. Oxford: Oxford University Press, 1961.

9. Kass RE and Raftery AE. Bayes factors. J Am Stat Assoc 1995; 90: 773-795.

10. Lee MD and Wagenmakers E-J. Bayesian cognitive modeling: a practical course. Cambridge: Cambridge University Press, 2014.

11. Schönbrodt FD and Wagenmakers EJ. Bayes factor design analysis: planning for compelling evidence. Psychon Bull Rev 2018; 25: 128-142.

12. Rouder JN. Optional stopping: no problem for Bayesians. Psychon Bull Rev 2014; 21: 301-308.

13. Schönbrodt FD, Wagenmakers E-J, Zehetleitner M, et al. Sequential hypothesis testing with Bayes factors: efficiently testing mean differences. Psychol Methods 2017; 22: 322

14. Dienes Z. Using Bayes to get the most out of nonsignificant results. Front Psychol 2014; 5: 781.

15. Wrinch D and Jeffreys H. XLII. On certain fundamental principles of scientific inquiry. London, Edinburgh, Dublin Philos Mag J Sci 1921; 42: 369-390.

16. Robert CP and Caron N. Noninformative Bayesian testing and neutral Bayes factors. Test 1996; 5: 411-437.

17. Benjamin DJ, Berger JO, Johannesson M, et al. Redefine statistical significance. Nat Hum Behav 2017; 2: 6-10.

18. Stefan AM, Gronau QF, Schönbrodt FD, et al. A tutorial on Bayes factor design analysis using an informed prior. Behav Res Methods 2019; 51: 1042-1058.

19. Gronau QF, Ly A and Wagenmakers EJ. Informed Bayesian t-tests. Am Stat 2020; 74: 137-143.

20. Gönen M, Johnson WO, Lu Y, et al. The Bayesian twosample t test. Am Stat 2005; 59: 252-257.

21. Ly A, Verhagen $J$ and Wagenmakers EJ. Harold Jeffreys's default Bayes factor hypothesis tests: explanation, extension, and application in psychology. $J$ Math Psychol 2016; 72: 19-32.
22. Rouder JN, Speckman PL, Sun D, et al. Bayesian t tests for accepting and rejecting the null hypothesis. Psychon Bull Rev 2009; 16: 225-237.

23. Mc Mahon B, Andersen SB, Madsen MK, et al. Seasonal difference in brain serotonin transporter binding predicts symptom severity in patients with seasonal affective disorder. Brain 2016; 139: 1605-1614.

24. Griffioen G, Matheson GJ, Cervenka S, et al. Serotonin 5-HT1A receptor binding and self-transcendence in healthy control subjects - a replication study using Bayesian hypothesis testing. PeerJ 2018; 6: e5790.

25. Plavén-Sigray P, Matheson GJ, Collste K, et al. Positron emission tomography studies of the glial cell marker translocator protein in patients with psychosis: a metaanalysis using individual participant data. Biol Psychiatry 2018; 84: 433-442.

26. Varnäs K, Cselényi Z, Jucaite A, et al. PET imaging of [11 C] PBR28 in Parkinson's disease patients does not indicate increased binding to TSPO despite reduced dopamine transporter binding. Eur J Nucl Med Mol Imaging 2018; 46: 367-375.

27. Dienes Z. Understanding psychology as a science: an introduction to scientific and statistical inference. London: Macmillan International Higher Education, 2008.

28. Gelman A, Stern HS, Carlin JB, et al. Bayesian data analysis. Boca Raton: Chapman and Hall/CRC, 2013.

29. Sanborn AN and Hills TT. The frequentist implications of optional stopping on Bayesian hypothesis tests. Psychon Bull Rev 2014; 21: 283-300.

30. Yu EC, Sprenger AM, Thomas RP, et al. When decision heuristics and science collide. Psychon Bull Rev 2014; 21 : 268-282.

31. Cohen J. Statistical power analysis for the behavioral sciences. London: Routledge, 1998.

32. Lakens D. Calculating and reporting effect sizes to facilitate cumulative science: a practical primer for t-tests and ANOVAs. Front Psychol 2013; 4: 863.

33. Nord M, Finnema SJ, Schain M, et al. Test-retest reliability of [11C]AZ10419369 binding to 5-HT 1B receptors in human brain. Eur J Nucl Med Mol Imaging 2014; 41: 301-307.

34. Collste K, Forsberg A, Varrone A, et al. Test-retest reproducibility of $[11 \mathrm{C}]$ PBR28 binding to TSPO in healthy control subjects. Eur J Nucl Med Mol Imaging 2016; 43: 173-183.

35. Finnema SJ, Nabulsi NB, Mercier J, et al. Kinetic evaluation and test-retest reproducibility of [11C]UCB-J, a novel radioligand for positron emission tomography imaging of synaptic vesicle glycoprotein $2 \mathrm{~A}$ in humans. $J$ Cereb Blood Flow Metab 2018; 38: 2041-2052.

36. Svensson JE, Schain M, Plavén-Sigray P, et al. Validity and reliability of extrastriatal [11C] raclopride binding quantification in the living human brain. Neuroimage 2019; 202: 116143.

37. Morey RD and Rouder JN. BayesFactor: Computation of Bayes Factors for Common Designs (v.0.9.12). The Comprehensive R Archive Network, 2018.

38. Parsey RV, Ogden RT, Miller JM, et al. Higher serotonin $1 \mathrm{~A}$ binding in a second major depression cohort: 
modeling and reference region considerations. Biol Psychiatry 2010; 68: 170-178.

39. Chen Y, Goldsmith J and Ogden T. Nonlinear mixedeffects models for PET data. IEEE Trans Biomed Eng 2019; 66: 881-891.

40. Schulz KF and Grimes DA. Multiplicity in randomised trials II: subgroup and interim analyses. Lancet 2005; 365: 1657-1661.

41. Proschan MA, Lan KKG and Wittes JT. Statistical monitoring of clinical trials: a unified approach. New York: Springer Science \& Business Media, 2006.

42. Lakens D. Performing high-powered studies efficiently with sequential analyses. Eur J Soc Psychol 2014; 44: 701-710.

43. Lachin JM. A review of methods for futility stopping based on conditional power. Stat Med 2005; 24: 2747-2764.

44. van 't Veer AE and Giner-Sorolla R. Pre-registration in social psychology - a discussion and suggested template. J Exp Soc Psychol 2016; 67: 2-12.
45. Poldrack RA, Baker CI, Durnez J, et al. Scanning the horizon: towards transparent and reproducible neuroimaging research. Nat Rev Neurosci 2017; 18: 115.

46. Knudsen GM, Ganz M, Appelhoff S, et al. Guidelines for the content and format of PET brain data in publications and archives: a consensus paper. J Cereb Blood Flow Metab 2020; 40: 1576-1585.

47. Quintana DS and Williams DR. Bayesian alternatives for common null-hypothesis significance tests in psychiatry: a non-technical guide using JASP. BMC Psychiatry 2018; 18: 178 .

48. van Doorn J, van den Bergh D, Bohm U, et al. The JASP guidelines for conducting and reporting a Bayesian analysis. Theor Rev. Epub ahead of print 9 October 2020. DOI: $10.3758 / \mathrm{s} 13423-020-01798-5$.

49. Love J, Selker R, Marsman M, et al. JASP: Graphical statistical software for common statistical designs. J Stat Softw 2019; 88: 1-17. 